Review

\title{
Current Approaches and Challenges in Managing and Monitoring Treatment Response in Ovarian Cancer
}

\author{
Charlotte S Marcus ${ }^{1,2}$, G Larry Maxwell1,3, Kathleen M Darcy¹, Chad A Hamilton ${ }^{1,2}$, William P McGuire ${ }^{\natural}$ \\ 1. Department of Defense Gynecologic Cancer Center of Excellence, Women's Health Integrated Research Center at Inova Health System, \\ Annandale, VA 22003, USA \\ 2. Gynecologic Oncology Service, Walter Reed National Military Medical Center, Bethesda, MD 20889, USA \\ 3. Department of Obstetrics and Gynecology, Inova Fairfax Hospital, Falls Church, VA22042, USA \\ 4. Department of Medicine, Inova Fairfax Hospital, Falls Church, VA 22042, USA
}

$\square$ Corresponding author: William P McGuire, Inova Medical Group - Oncology, 3289 Woodburn Road, Suite 320, Annandale, VA 22003, William.McGuire@inova.org; 703-970-6545

( ) Ivyspring International Publisher. This is an open-access article distributed under the terms of the Creative Commons License (http://creativecommons.org/ licenses/by-nc-nd/3.0/). Reproduction is permitted for personal, noncommercial use, provided that the article is in whole, unmodified, and properly cited.

Received: 2013.11.01; Accepted: 2013.11.25; Published: 2014.01.01

\begin{abstract}
Epithelial ovarian cancer is the leading cause of death among gynecologic malignancies. Treatment of recurrent ovarian cancer remains a challenge despite advances in surgical and chemotherapeutic options. A goal of many providers is to detect recurrences as early as possible and initiate treatment though there is controversy as to whether this impacts outcome. Elevations in CAI25 and radiological findings may precede symptoms of recurrence by several months. While detection of recurrences by physical exam alone is unusual, a thorough exam in conjunction with reported symptoms and elevated CAI 25 is sufficient to detect $80-90 \%$ of recurrences. A spiral CT scan may be used to confirm recurrence in the setting of asymptomatic CAI25 elevation and a $\mathrm{PET} / \mathrm{CT}$ can yield additional insight if the $\mathrm{CT}$ is inconclusive. Initiating chemotherapy prior to the development of symptoms, even in the setting of elevated CAI25, does not impact overall survival primarily because the efficacy of available treatments in the recurrent setting is poor. More information about tumor biology and ways to predict which patients will benefit from available treatment options is required. Consequently, the approach to post-treatment surveillance should be individualized taking into account the clinical benefit of the second-line therapy, versus the costs and morbidity of the surveillance method.
\end{abstract}

Key words: Ovarian Cancer, Treatment Response

\section{Introduction}

According to SEER data 22,240 women will be diagnosed with ovarian cancer and approximately 14,000 women will die of this disease in 2013 [1, 2]. Although relatively rare ovarian cancer is characterized by a 5 year survival of $44.2 \%$ since greater than $75 \%$ of women present with advanced disease [3]. Approximately $75 \%$ of these patients will have a complete clinical response. Among those patients who are stage III and optimally debulked, 50\% will have a complete pathological response after first line chemotherapy with a median progression free sur- vival of 18 months [4]. Roughly 20 to $30 \%$ of these women will progress or fail to achieve a complete clinical response while receiving first-line therapy and are classified as platinum refractory. Another $25 \%$ of women will relapse within 6 months after completion of first-line therapy and are classified as platinum resistant. The remaining patients are considered platinum sensitive and are retreated with platinum-based therapies increasing the risk of platinum related cumulative toxicities.

Many providers focus on detection of recurrence 
as early as possible and aggressive treatment of these recurrences based on the premise that this will improve outcomes. There are many options for surveillance of ovarian cancer including physical exams, various imaging modalities, and measurement of serum tumor markers. Significant controversy exists as to whether any of these modalities ultimately affect patient survival [5-8]. Von Georgi et al studied 704 patients who had no evidence of disease after standard adjuvant therapy and were followed by a variety of surveillance modalities, none of which made a difference in survival [5]. Elevations of CA125 may, for example, antedate patient symptoms by weeks to months calling into question the definition of when relapse has occurred [9]. Modalities that are used to define progression free survival in the research setting may have limited utility in the clinical setting. Although there are several options for salvage treatment, these agents will offer palliation, and may extend disease control and survival but are rarely curative [9]. Ten-year survival rates for women diagnosed with advanced stage disease and treated first-line with intravenous platinum and taxane combination chemotherapy remains about $10 \%$. Patients and physicians must carefully consider which of these surveillance options to pursue given limited efficacy, impact on quality of life, and often limited resources. The estimated costs per patient recurrence based on Southern California Medicare data were approximately $\$ 42,000$ for exams, $\$ 4000$ for serum CA125, and $\$ 13,000$ for CT scans [10]. The available modalities for surveillance and benefits and risks for each will be reviewed.

\section{Current recommendations}

The National Comprehensive Cancer Network (NCCN) and Society of Gynecologic Oncology (SGO) both have recommendations for ovarian cancer surveillance. The NCCN recommends serial visits including pelvic exams and measurement of CA-125 if initially elevated. Imaging is recommended if clinically indicated with PET scans receiving a Category 2B recommendation (i.e. NCCN consensus based on retrospective studies). The remainder of evidence for the NCCN guidelines is Category 2A or uniform consensus based on "low-level" or retrospective studies that the intervention is appropriate. In June 2011, SGO published recommendations for post-treatment surveillance in women who had achieved a full response to adjuvant therapy. These evidence-based guidelines are generally congruent with the NCCN guidelines with an emphasis on symptom assessment and the physical exam. According to SGO recommendations, the role of CA125 should be reviewed with patients and surveillance with this marker is optional [3].

\section{Physical Exam}

The follow up visit and physical exam is the cornerstone of post-treatment surveillance with visits recommended every 2-4 months for two years then every 4-6 months for three years then annually after five years. Chan et al (2008) conducted a retrospective study to determine how many recurrences were detected based on physical exam, imaging, or CA125 level [11]. Out of 80 patients three (4\%) first presented with physical findings while 28 (35\%) first presented with symptoms [11]. CA125 was elevated in over $90 \%$ of patients presenting with recurrences and, after further questioning or more detailed physical exams, symptoms and physical findings were detected [11]. Consequently, among patients who recurred 55\% had symptoms and $53 \%$ had physical findings [11]. Patients who had an elevated CA-125 had significantly worse survival than patients with normal values. However, when patients with elevated CA125 were stratified by the presence of symptoms or abnormal physical findings there was no difference in survival[11]. A study by Fehm et al (2005) in 58 patients with recurrent ovarian cancer found that $60 \%$ of patients presented with symptoms, the most common of which was abdominal pain [12]. Further $77 \%$ had evidence of recurrence on physical exam, $89 \%$ in cases of pelvic recurrence, while $83 \%$ had an elevated CA125. It is not clear from this study whether the patients first presented with physical findings or whether the findings were discovered after elevated CA125 or the patient's complaints [12]. Von Georgi et al reported in their cohort of 704 ovarian cancer patients that $28 \%$ of these women were diagnosed by symptoms, and $15 \%$ by gynecological exam [5]. In many of these studies the physical examinations were performed by trainees and general gynecologists which may have affected the rate of detection. However, these results suggest the performance of a physical exam should be driven by either patient symptoms or elevated CA125 because detection by physical exam alone is rare $[10,11,13]$. On a cost basis, physical exam is associated with the highest cost per patient recurrences [10]. Lastly, though not routinely performed in most centers, the sensitivity of vaginal cytology is also poor for detecting recurrence and not recommended in current guidelines [10].

\section{Imaging}

The Response Evaluation Criteria in Solid Tumors (RECIST) are used to assess tumor response in both trials and clinical settings [14]. These criteria were developed to standardize how response is measured and simplify tumor measurements using unidimensional parameters versus the product of 
perpendicular diameters. The GCIG criteria for progression by RECIST are any new lesion or a $20 \%$ increase in the sum of the longest diameters of all lesions. These progression criteria may be used alone or in combination with CA125 for evaluating new drugs $[15,16]$. Independent reviews of objective response based on imaging in ovarian cancer are unable to be confirmed in $30-40 \%$ of cases [17-19]. Hopper et al (1996) studied the interobserver reliability among three radiologists evaluating tumor measurements on thoracic and abdominopelvic CT scans. Radiologists were first asked to identify the indicator lesion (i.e. most representative of tumor burden) and then asked to measure the size of other independent foci. Approximately $25 \%$ of the time there was agreement among two or three radiologists about the indicator lesion and there was $15 \%$ interobserver variability in overall CT measurement [18]. In a study by Muenzel, the variability in the change of the longest diameter was $24 \%$ resulting in misclassification of response in half of the cases. Accuracy was improved when the same radiologist read the initial and follow-up studies, or when multiple readers reviewed the images for consensus [20]. Further, the percent of patients classified as complete responders decreased from $26 \%$ to $15 \%$ after an independent review of imaging subsequent to a trial of second line agents in ovarian cancer [19]. In a multivariate analysis of prognostic indicators, CA-125 performed twice as well as RECIST in predicting survival [21]. Prior studies have shown that radiological progression may precede clinical progression by 2-3 months and, when combined with CA125, can precede clinical progression by almost five months [22]. Moreover, there remains a subset of patients without initial elevation in CA125 or medical conditions affecting CA125 level (e.g. abdominal surgery or peritonitis) where imaging and relapse based on RECIST criteria will be the standard method of follow up [23].

Although there are many imaging options for surveillance, multiple studies have shown no overall survival benefit to serial radiologic studies on [3, 12]. Fehm et al reported combined vaginal and abdominal ultrasound revealed recurrence in $70 \%$ of patients but was less sensitive than vaginal exam in patients with pelvic recurrence [12]. In 17/58 patients who did not have a pelvic recurrence, nothing was seen on ultrasound [12]. In a series of 83 patients with recurrence reviewed by Testa et al, the positive predictive value was $100 \%$ but ultrasound failed to detect 22 cases of recurrences in this series [24]. The reported sensitivity of ultrasound is $45 \%$ to $85 \%$ and specificity is $60-100 \%$ [25]. Real-time ultrasound findings also may be different than still images that are capturing a limited amount of data for review by investigators.
Computed tomography (CT) scan aids in the evaluation of patients with asymptomatic recurrence and in the planning of secondary cytoreductive surgery. Conventional CT has limited sensitivity of $40-93 \%$ and specificity of 50-98\% for recurrent disease $[3,25]$. Spiral CT has a higher sensitivity (particularly for peritoneal metastases) than conventional CT which detects $50-67 \%$ of peritoneal lesions and implants. Lesions that are surrounded by ascites are more readily detectable and lead to less false negatives than lesions without proximal ascites. Obtaining a CT prior to secondary debulking may aid in surgical planning since hydronephrosis and invasion into the pelvic sidewall are strong indicators of non-resectability [26]. In terms of cost effectiveness, $60-70 \%$ of recurrences are detected with CT scan at a cost of $\$ 13,454$ per recurrent diagnosis. The sensitivity of MRI in detecting recurrences is similar to that of CT scan in lesions greater than $2 \mathrm{~cm}$, however, MRI is especially useful in the detection of lesions on peritoneal surfaces and bowel serosa, the vaginal vault, cul-de-sac, and bladder base or if patients cannot have a contrast-enhanced CT $[25,27,28]$. CT remains the first choice modality over MRI because it is more widely available and less costly to obtain than an MRI.

Positron emission tomography (PET)/CT using fluoro-2-deoxy-D-glucose (FDG-PET/CT) FDG- performs better than CT and MRI, particularly in the setting of suspected recurrence $[25,28]$. Clinical experience also shows PET/CT aids in planning of secondary cytoreduction by identifying those patients with unresectable disease $[27,29,30]$. Thrall et al conducted a retrospective chart review of 29 ovarian cancer patients who had inconclusive CT scans and a rising CA125 level and reported a sensitivity of $94.5 \%$, a specificity of $100 \%$ for PET/CT in detecting recurrent disease and more precise localization versus CT scan alone [29]. In another series of 66 patients, conventional and PET CT detected recurrence in approximately the same number of patients with symptoms but a normal CA125. However, $31 \%$ of patients with no evidence of disease on CT scan had lesions present on PET/CT [31]. Bristow investigated the ability of PET/CT to predict macroscopic disease at time of secondary debulking in 22 patients with epithelial ovarian cancer and rising CA125. PET/CT accurately detected recurrence in slightly more than $80 \%$ of patients [32]. The tumor size for the 18 out of 22 patients who recurred ranged from 1.5 to $3.2 \mathrm{~cm}$ (median $2.3 \mathrm{~cm}$ ). This finding was supported in subsequent studies showing inability to detect small volume disease $<1 \mathrm{~cm}$ with PET/CT [27]. Therefore, a CT scan should be performed in patients with a rising CA125 or symptoms suspicious for recurrence. If the CT scan is inconclusive or if the patient is a good candidate for 
secondary cytoreduction a PET/CT will offer additional insights.

\section{CA125}

Cancer antigen 125 or CA125 is a glycoprotein that is the current standard of care biomarker for ovarian cancer surveillance. However, this approach is not without controversy in terms of its role in cancer screening or the effect of CA125 surveillance on long term survival. CA125 is elevated in $69 \%-88 \%$ of ovarian cancers depending on histology; it is elevated in $80 \%$ of serous cancers [33]. Unfortunately, it is elevated in only $50 \%$ of stage I ovarian cancers and therefore, has limited utility as a biomarker for screening since it is the patient with stage I disease that is most amenable to surgical cure [22]. The sensitivity of CA125 for recurrence is $62-94 \%$ and the specificity is $91-100 \%[25,34]$. The CA125 level rises at least 3 months before recurrence and there is a median lag time of two months between the elevation in CA125 and findings on imaging studies $[35,36]$. The GCIG defines recurrent ovarian cancer as elevation of CA125 $\geq$ twice the upper limit of normal on two occasions at least one week apart in those patients who had an elevated marker at diagnosis with normalization after treatment [16]. The same criteria are used for patients who did not have an elevated CA125 pretreatment. If CA125 was initially elevated but did not normalize, progressive disease is defined by CA125 $\geq$ twice the nadir value on two occasions at least one week apart. These criteria were developed for patients on cytotoxic chemotherapy but have not yet been validated in patients on targeted therapies [16, 37]. Non-specific elevations in CA125 may be observed in patients with manipulation of pleura or peritoneum within the last 28 days [30]. There is a high false negative rate as $50 \%$ of patients with normal CA125 at the conclusion of primary therapy have microscopic disease with second-look surgery [22]. The cost per recurrence of obtaining CA125 according to the currently recommended schedule is approximately $\$ 3924$ per patient recurrence [10].

Rustin et al. reported results of a landmark prospective trial that called into question the use of CA125 in surveillance of ovarian cancer [38]. This trial by the UK Medical Research Council and the European Organization for the Research and Treatment of Cancer (MRC OV05/EORTCC 55955) entered 529 women in complete remission into a randomized trial of early treatment based solely upon a rising CA125 but no symptoms versus treatment of symptomatic recurrence without regard to CA125 levels. In the latter group, physicians were blinded to the CA125 levels. Data on the extent of their initial surgery was not available. Patients also completed a quality of life questionnaire prior to starting each chemotherapy cycle. The median survival in patients assigned to early therapy was 25.7 months versus 27.1 months in those receiving delayed therapy (NS). Analysis of treatment interactions (e.g. age, and type of second line chemotherapy) did not reveal any skewing of factors that may have influenced outcome. Women who were in the early group had earlier deterioration in quality of life versus women in the delayed treatment group. There are several issues with this study that limit its applicability to current clinical practice including changes in second-line chemotherapy options (i.e. biologics or liposomal doxorubicin which have less effect on quality of life) during the period of accrual (i.e. 9 years) and completion. Moreover, only $7 \%$ of patients in this study underwent secondary debulking surgery which may have altered outcome or quality of life and was more likely to be feasible in those patients diagnosed early [39]. The risks and benefits of this procedure is beyond the scope of this review; however, there are several lines of evidence that suggest a survival benefit for the carefully selected patient that is optimally cytoreduced and resumes platinum-based chemotherapy [40,41]. Further Morris argues that there may have been a selection bias in favor of patients with a worse prognosis since $54 \%$ of patients had a platinum-free interval less than a year and physicians may not have enrolled patients with better prognosis into the study for concern of randomization to the delayed treatment arm.

Another point of controversy relates to the definitive CA125 level that is used to indicate recurrence and differences in prognosis based on the variations within the normal range [25, 42-45]. Several authors argue that the GCIG criteria are too strict and that using this criterion alone has low sensitivity. Prat et al reported that if the nadir CA125 is in the normal range, an increase of $\geq 5 \mathrm{U} / \mathrm{ml}$ compared with the nadir value predicts recurrence with $90 \%$ sensitivity with a $96.4 \%$ positive predictive value [44]. Further Liu et al suggest that for patients with CA125 s $10 \mathrm{U} / \mathrm{ml}$ and elevation to $\geq 20 \mathrm{U} / \mathrm{ml}$ is predictive of recurrence. If patients had a CA125 $>10 \mathrm{U} / \mathrm{ml}$ after therapy, at least doubling or the nadir value is predictive of recurrence. Markman et al also validated other reports that a post-treatment CA125 level of 10-12 $\mathrm{U} / \mathrm{ml}$ offers a significant survival advantage. In this study the median PFS was 24,17 , and 7 months if the pre-maintenance CA125 was $\leq 10 \mathrm{U} / \mathrm{ml}, 11-20$ $\mathrm{U} / \mathrm{ml}$, and 21 to $35 \mathrm{U} / \mathrm{ml}$ respectively [45]. Xu et al reported similar findings in a less heterogeneous cohort of 616 patients with high grade serous ovarian cancer confirmed with pathology review. Further, whereas other authors used an arbitrary CA125 value, the median CA125 nadir in this study was $10 \mathrm{U} / \mathrm{ml}$. 
Interestingly, in a subset of 80 patients undergoing second look surgery, there was no correlation between the tumor burden and the CA125 nadir possibly due to small cohort size or the inability of CA125 to discriminate with low tumor burden. The distribution of nadir CA125 within the normal range in MRC OV05/EORTCC 55955 is not reported and the GCIG criteria for CA125 progression were used. The patients started second-line chemotherapy an average of five months after randomization. Until prospective data is available to corroborate this practice, initiation of treatment based on a lower CA125 value would allow for a longer lead time, longer duration on salvage treatment with more toxicity, and increased patient anxiety without any benefit since there is no evidence of increased survival when initiating treatment before presentation of symptoms [38].

Questions also remain around utilization of CA125 in patients who are treated and exposed to mouse monoclonal antibodies. This is particularly important in light of new agents that exploit this technology. CA125 and other in vitro assays are known to be unreliable in the presence of human anti-mouse antibodies (HAMA) which are generated in response to treatment with murine antibodies [46, 47]. However, most of the commercially available antibodies, e.g. bevacizumab, are fully humanized and there is no production of HAMA. The newer CA125 diagnostic tests are also able to achieve accurate measurements by filtering out the HAMA antibodies. Still there is the issue of the performance of serum CA125 because biologic therapies may alter the tumor's production of mucin MUC16 that is recognized by an antibody in the CA125 assay [48]. Randall et al compared the response classification by radiographic RECIST versus CA125 criteria in a follow-on study to GOG 170-D [37]. CA125 was collected as part of the study but not used to define progression initially in the sixty-two evaluable patients. The median PFS in this case by RECIST criteria was 4.7 months and 5.6 months by CA125 which is fairly similar. However 8 of the 62 patients were identified as having progressed based on CA125 level from 6 to 38 months before RECIST-defined progression [37]. Azad et al also investigated the use of CA125 compared with RECIST criteria in patients treated with sorafenib and bevacizumab in a small study of 15 patients [48]. There was a $67 \%$ concordance between CA125 criteria and objective imaging defining response. Three patients were classified with progressive disease by CA125 criteria whereas objective imaging classified these patients as partial responders. In both studies, patients would have been prematurely removed from the therapies based on the CA 125 levels obtained. The emergence of an increasing number of molecularly targeting agents developed for use as single agents or in combination with cytotoxic and/or cytostatic drugs prompts the need for additional research to refine the clinical utility of CA125 in monitoring ovarian cancer recurrence and disease progression and may lead to further modifications to RECIST.

\section{Conclusion}

The majority of ovarian cancer patients will relapse within five years requiring salvage chemotherapy. Current practice involves long-term surveillance of women for recurrence by exams, serum biomarkers, and imaging. There is no evidence that any of these modalities will impact survival versus waiting for the presentation of symptoms and initiating treatment at that point in time. However, for many patients the follow-up visit presents an opportunity for medical reassurance about the likelihood of recurrence despite counseling about the limitations of the screening tests [49]. Furthermore, patients with a rising CA125 may suffer anxiety knowing that their disease is recurring but treatment is not recommended until they have symptoms. The psychological benefit to follow up is important, and providing these services in a cost-effective manner is paramount. Interventions to proactively reduce patient stress, anxiety and/or depression may be an appropriate consideration. The approach to post-treatment surveillance should be individualized taking into account the clinical benefit of the second-line therapy, costs, morbidity and mortality of the surveillance methods, available treatment options and, lastly, patient preference [5].

\section{Disclaimer}

The opinions or assertions contained herein are the private views of the authors and are not to be construed as official or as reflecting the views of the Department of the Army, the Department of the Air Force, the Department of the Navy, or the Department of Defense.

\section{Competing Interests}

The authors have declared that no competing interest exists.

\section{References}

1. [Internet] Howlader N NA, Krapcho M, Garshell J, Neyman N, Altekruse SF, Kosary CL, Yu M, Ruhl J, Tatalovich Z, Cho H, Mariotto A, Lewis DR, Chen HS, Feuer EJ, Cronin KA. SEER Cancer Statistics Review, 1975-2010. http://seercancergov/csr/1975_2010/

2. Siegel R, Naishadham D, Jemal A. Cancer statistics, 2013. CA: a cancer journal for clinicians. 2013; 63: 11-30.

3. Salani R, Backes FJ, Fung Kee Fung M, Holschneider CH, Parker LP, Bristow $\mathrm{RE}$, et al. Posttreatment surveillance and diagnosis of recurrence in women with gynecologic malignancies: Society of Gynecologic Oncologists recommendations. American Journal of Obstetrics and Gynecology. 2011; 204: 466-78. 
4. McGuire WP, Hoskins WJ, Brady MF, Kucera PR, Partridge EE, Look KY, et al. Cyclophosphamide and Cisplatin Compared with Paclitaxel and Cisplatin in Patients with Stage III and Stage IV Ovarian Cancer. New England Journal of Medicine. 1996; 334: 1-6.

5. von Georgi R, Schubert K, Grant P, Munstedt K. Post-therapy surveillance and after-care in ovarian cancer. European journal of obstetrics, gynecology, and reproductive biology. 2004; 114: 228-33.

6. Geurts SM, de Vegt F, van Altena AM, Tjan-Heijnen VC, Massuger LF, Adang $\mathrm{EM}$, et al. Impact of routine follow-up examinations on life expectancy in ovarian cancer patients: a simulation study. International journal of gynecological cancer : official journal of the International Gynecological Cancer Society. 2012; 22: 1150-7.

7. Kew F, Galaal K, Bryant A, Naik R. Evaluation of follow-up strategies for patients with epithelial ovarian cancer following completion of primary treatment. Cochrane database of systematic reviews (Online). 2011;: Cd006119.

8. Miller RE, Rustin GJ. How to follow-up patients with epithelial ovarian cancer. Current opinion in oncology. 2010; 22: 498-502.

9. Armstrong DK. Relapsed Ovarian Cancer: Challenges and Management Strategies for a Chronic Disease. The Oncologist. 2002; 7: 20-8.

10. Rettenmaier NB, Rettenmaier CR, Wojciechowski T, Abaid LN, Brown JV III, Micha JP, et al. The utility and cost of routine follow-up procedures in the surveillance of ovarian and primary peritoneal carcinoma: a 16-year institutional review. Br J Cancer. 2010; 103: 1657-62.

11. Chan KKL, Tam KF, Tse KY, Ngan HYS. The role of regular physical examination in the detection of ovarian cancer recurrence. Gynecologic Oncology. 2008; 110: 158-61.

12. Fehm T, Heller F, Kramer S, Jager W, Gebauer G. Evaluation of CA125, physical and radiological findings in follow-up of ovarian cancer patients. Anticancer research. 2005; 25: 1551-4.

13. Menczer J, Chetrit A, Sadetzki S, Golan A, Levy T. Follow-up of ovarian and primary peritoneal carcinoma: The value of physical examination in patients with pretreatment elevated CA125 levels. Gynecologic Oncology. 2006; 103: 137-40.

14. Therasse P, Arbuck SG, Eisenhauer EA, Wanders J, Kaplan RS, Rubinstein L, et al. New guidelines to evaluate the response to treatment in solid tumors. European Organization for Research and Treatment of Cancer, National Cancer Institute of the United States, National Cancer Institute of Canada. Journal of the National Cancer Institute. 2000; 92: 205-16.

15. Eisenhauer EA. Optimal assessment of response in ovarian cancer. Annals of oncology : official journal of the European Society for Medical Oncology / ESMO. 2011; 22 Suppl 8: viii49-viii51.

16. Rustin GJ, Vergote I, Eisenhauer E, Pujade-Lauraine E, Quinn M, Thigpen T, et al. Definitions for response and progression in ovarian cancer clinical trials incorporating RECIST 1.1 and CA 125 agreed by the Gynecological Cancer Intergroup (GCIG). International journal of gynecological cancer : official journal of the International Gynecological Cancer Society. 2011; 21: 419-23.

17. Markman M. The Myth of Measurable Disease in Ovarian Cancer. Journal of Clinical Oncology. 2003; 21: 3013-5.

18. Hopper KD, Kasales CJ, Van Slyke MA, Schwartz TA, TenHave TR, Jozefiak JA. Analysis of interobserver and intraobserver variability in CT tumor measurements. AJR American journal of roentgenology. 1996; 167: 851-4.

19. Gwythen S, Bolis G, Gore M, Huinink WtB, Verweij J, Hudson IR, et al. Experience with independent radiological review during a topotecan trial in ovarian cancer. Annals of Oncology. 1997; 8: 463-8.

20. Muenzel D, Engels HP, Bruegel M, Kehl V, Rummeny EJ, Metz S. Intra- and inter-observer variability in measurement of target lesions: implication on response evaluation according to RECIST 1.1. Radiology and oncology. 2012; 46: 8-18.

21. Grolund B HC, Hilden J, Engelholm SA, Hogdall EV, Hansen HH. Should CA-125 response criteria be preferred to response evaluation criteria in solid tumores (RECIST) for prognostication during second-line chemotherapy of ovarian carcinoma? J Clin Oncol. 2004; 22: 4051-8.

22. Pignata S, Cannella L, Leopardo D, Bruni GS, Facchini G, Pisano C. Follow-up with CA125 after primary therapy of advanced ovarian cancer: in favor of continuing to prescribe CA125 during follow-up. Annals of oncology : official journal of the European Society for Medical Oncology / ESMO. 2011; 22 Suppl 8: viii40-viii4.

23. Rustin GJS. Can We Now Agree to Use the Same Definition to Measure Response According to CA-125? Journal of Clinical Oncology. 2004; 22: 4035-6.

24. Testa AC, Fruscella E, Ludovisi M, De Vincenzo R, Malaggese M, Corrado G, et al. The role of sonographic examination in the follow-up of gynecological neoplasms. Gynecol Oncol. 2005; 99: 696-703.

25. Gadducci A, Cosio S. Surveillance of patients after initial treatment of ovarian cancer. Critical reviews in oncology/hematology. 2009; 71: 43-52.

26. Stacey A. Funt HH, Nadeem Abu-Rustum, Madhu Mazumdar, Howard Felderman and Dennis S. Chi. Role of CT in the Management of Recurrent Ovarian Cancer. American Journal of Roentgenology. 2004; 182: 393-8.

27. Bharwani N, Reznek RH, Rockall AG. Ovarian Cancer Management: the role of imaging and diagnostic challenges. European journal of radiology. 2011; 78: 41-51.

28. Javitt MC. ACR Appropriateness Criteria on staging and follow-up of ovarian cancer. Journal of the American College of Radiology : JACR. 2007; 4: 586-9.

29. Thrall MM, DeLoia JA, Gallion H, Avril N. Clinical use of combined positron emission tomography and computed tomography (FDG-PET/CT) in recurrent ovarian cancer. Gynecol Oncol. 2007; 105: 17-22.
30. Colombo N, Peiretti M, Parma G, Lapresa M, Mancari R, Carinelli S, et al. Newly diagnosed and relapsed epithelial ovarian carcinoma: ESMO Clinical Practice Guidelines for diagnosis, treatment and follow-up. Annals of Oncology. 2010; 21: v23-v30.

31. Bhosale P, Peungjesada S, Wei W, Levenback CF, Schmeler K, Rohren E, et al. Clinical utility of positron emission tomography/computed tomography in the evaluation of suspected recurrent ovarian cancer in the setting of normal CA-125 levels. International journal of gynecological cancer : official journal of the International Gynecological Cancer Society. 2010; 20: 936-44.

32. Bristow RE, del Carmen MG, Pannu HK, Cohade C, Zahurak ML, Fishman EK, et al. Clinically occult recurrent ovarian cancer: patient selection for secondary cytoreductive surgery using combined PET/CT. Gynecol Oncol. 2003; 90: 519-28.

33. Su Z, Graybill WS, Zhu Y. Detection and monitoring of ovarian cancer. Clinica chimica acta, international journal of clinical chemistry. 2013; 415: 341-5.

34. Song MJ, Lee SH, Choi MR, Son HJ, Lee CW, Yoon JH, et al. Diagnostic value of CA125 as a predictor of recurrence in advanced ovarian cancer. European journal of gynaecological oncology. 2013; 34: 148-51.

35. Gadducci A, Sartori E, Zola P, Landoni F, Maggino T, Palai N, et al. Serum CA125 assay in the early diagnosis of recurrent epithelial ovarian cancer. Oncology reports. 1996; 3: 301-3.

36. Bast RC, Jr., Xu FJ, Yu YH, Barnhill S, Zhang Z, Mills GB. CA 125: the past and the future. The International journal of biological markers. 1998; 13: 179-87.

37. Randall LM, Sill MW, Burger RA, Monk BJ, Buening B, Sorosky JI. Predictive value of serum CA-125 levels in patients with persistent or recurrent epithelial ovarian cancer or peritoneal cancer treated with bevacizumab on a Gynecologic Oncology Group phase II trial. Gynecol Oncol. 2012; 124: 563-8.

38. Rustin GJ, van der Burg ME, Griffin CL, Guthrie D, Lamont A, Jayson GC, et al. Early versus delayed treatment of relapsed ovarian cancer (MRC OV05/EORTC 55955): a randomised trial. Lancet. 2010; 376: 1155-63.

39. Tanner EJ, Chi DS, Eisenhauer EL, Diaz-Montes TP, Santillan A, Bristow RE. Surveillance for the detection of recurrent ovarian cancer: survival impact or lead-time bias? Gynecol Oncol. 2010; 117: 336-40.

40. Al Rawahi T LA, Bristow RE, Bryant A, Elattar A, Chattopadhyay S, Galaal K. Surgical cytoreduction for recurrent epithelial ovarian cancer. Cochrane database of systematic reviews (Online). 2013.

41. Lorusso D, Mancini M, Di Rocco R, Fontanelli R, Raspagliesi F. The Role of Secondary Surgery in Recurrent Ovarian Cancer. International Journal of Surgical Oncology. 2012; 2012: 6.

42. Crawford SM, Peace J. Does the nadir CA125 concentration predict a long-term outcome after chemotherapy for carcinoma of the ovary? Annals of Oncology. 2005; 16: 47-50.

43. Liu PY, Alberts DS, Monk BJ, Brady M, Moon J, Markman M. An early signal of CA-125 progression for ovarian cancer patients receiving maintenance treatment after complete clinical response to primary therapy. J Clin Oncol. 2007; 25: 3615-20.

44. Prat A, Parera M, Peralta S, Perez-Benavente MA, Garcia A, Gil-Moreno A, et al. Nadir CA-125 concentration in the normal range as an independent prognostic factor for optimally treated advanced epithelial ovarian cancer. Annals of Oncology. 2008; 19: 327-31.

45. Markman M, Liu PY, Rothenberg ML, Monk BJ, Brady M, Alberts DS. Pretreatment CA-125 and risk of relapse in advanced ovarian cancer. J Clin Oncol. 2006; $24: 1454-8$

46. Bertholf RL, Johannsen L, Guy B. False elevation of serum CA-125 level caused by human anti-mouse antibodies. Annals of clinical and laboratory science. 2002; 32: 414-8.

47. Oei ALM, Sweep FCGJ, Massuger LFAG, Olthaar AJ, Thomas CMG. Transient human anti-mouse antibodies (HAMA) interference in CA 125 measurements during monitoring of ovarian cancer patients treated with murine monoclonal antibody. Gynecologic Oncology. 2008; 109: 199-202.

48. Azad NS, Annunziata CM, Steinberg SM, Minasian L, Premkumar A, Chow C, et al. Lack of reliability of CA125 response criteria with anti-VEGF molecularly targeted therapy. Cancer. 2008; 112: 1726-32.

49. Bradley EJ, Pitts MK, Redman CW, Calvert E. The experience of long-term hospital follow-up for women who have suffered early stage gynecological cancer: a qualitative interview study. International journal of gynecological cancer : official journal of the International Gynecological Cancer Society. 1999; 9: 491-6. 